\title{
Bilanz ziehen:
}

\section{Zahlen, Daten}

und Fakten in der Pädiatrie

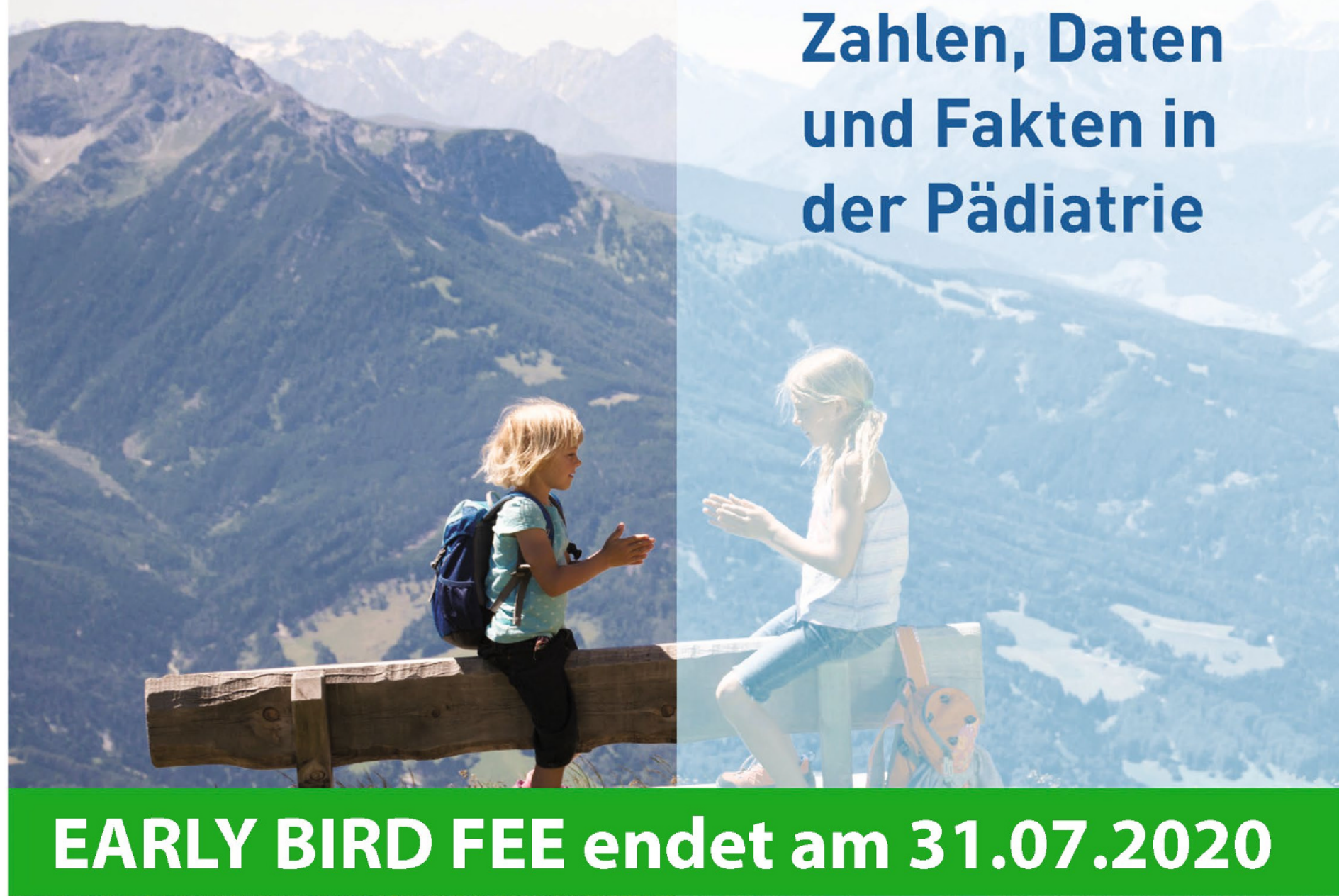

58. Jahrestagung der Österreichischen

Gesellschaft für

Kinder- und

Jugendheilkunde
24.-26.

September

2020

Congress

Innsbruck 


\section{Mitteilungen der ÖGKJ}

Monatsschr Kinderheilkd 2020 • 168:564-572

https://doi.org/10.1007/s00112-020-00930-y

(c) Springer Medizin Verlag GmbH, ein Teil von Springer Nature 2020

\section{Redaktion (V.i.S.d.P.)}

Prof. Dr. Reinhold Kerbl

LKH Hochsteiermark/Leoben

Abteilung für Kinder und Jugendliche

Vordernbergerstraße 42

A-8700 Leoben

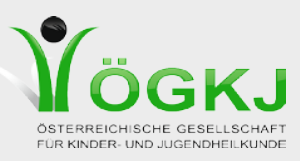

Präsidentin

Prof. Dr. Daniela Karall, Innsbruck

\section{Sekretärin}

PD Dr. Sabine Scholl-Bürgi, Innsbruck

Geschäftsstelle der ÖGKJ für Öffentlichkeitsarbeit und Standesführung

Ralph Kerschbaumer

c/o S12! studio12 gmbh

Kaiser Josef Straße 9, A-6020 Innsbruck

Tel.: +43512 890438 · Fax: + 43 512-890438-15

E-Mail: office@studio12.co.at·Internet: www.paediatrie.at

\section{SARS-CoV2, Corona, COVID19 und $\mathrm{CO} . .$.}

Liebe Kolleginnen und Kollegen!

Besondere Zeiten liegen hinter und vor uns - wenn diese Zeilen gedruckt und ausgesandt werden, sind sie möglicherweise nicht mehr aktuell oder relevant.

Trotzdem möchten wir in dieser Ausgabe der Mitteilungen der ÖGKJ in der Monatsschrift Kinderheilkunde einige Beiträge zu Corona-Themen bringen.

Für die ÖGKJ relevanteste Frage:

\section{WIRD DIE JAHRESTAGUNG HEUER STATTFINDEN?} JA!

Zum heutigen Stand (18.05.2020) gehen wir davon aus, dass die Jahrestagung wie vorgesehen, vom 24.09. bis 26.09.2020 in Innsbruck stattfinden wird, möglicherweise und vermutlich mit Beachtung einiger Maßnahmen, die wir bisher auch schon beachten mussten (z.B. Achten auf eine bestimmte maximale Anzahl der Teilnehmer pro Raum u.ä.). Die jeweils aktualisierten Informationen sind auf der Homepage www.oegkj-jahrestagung.at oder auch www.paediatrie.at zu finden.

Jedenfalls kann die Anmeldung derzeit regulär stattfinden! Die Early Bird Registratierung endet mit 31.07.2020.

Vielen Dank an alle, die in Krankenhäusern und Ordinationen und auch an anderen Stellen unter z. T. sehr ungewöhnlichen und mit nicht ganz klar definierten Rahmenbedinungen die Versorgung von Kindern und Jugendlichen gewährleistest und aufrecht erhalten haben! Das haben Sie alle sehr gut bewältigt und gemacht! Vielen Dank auch für all die hilfreichen Nachrichten und Beiträge und die Kommunikation in dieser besonderen Zeit!

Herzliche Grüße,

\section{Daniela Karall}

Sabine Scholl-Bürgi

Innsbruck, 30.04.2020

\section{Inhalt}

565 SARS-CoV2, Corona, COVID19 und co....

565 SARS-CoV2 Situation bei österreichischen Kindern (30.04.2020)

566 Statement zur Versorgung von Menschen mit seltenen und/oder chronischen Erkrankungen in Zeiten von COVID-19

568 Coronavirus/ COVID-19 und Stillen: Aktuelle internationale Empfehlungen

569 Schwangerschaft und Stillen in Zeiten von COVID19

571 4. Österreichische Jahrestagung für Pädiatrische Psychosomatik

571 Geburtstage

\section{SARS-CoV2 Situation bei österreichischen Kindern (30.04.2020)}

Rund um die Diskussion über die bevorstehenden Schulöffnungen sind die SARS-CoV2 Infektionszahlen bei Kindern zunehmend in das allgemeine Interesse gerückt. Bis Ende April 2020 waren in Österreich von 15.410 positiv mittels SARS-CoV2 PCR getesteten Personen 84 $(0,5 \%)$ unter 5 Jahre alt und 348 (2,3\%) zwischen 5 und 15 Jahren alt. Aus diesen von den Gesundheitsbehörden erhobenen und zur Verfügung gestellten Daten geht jedoch die Indikation für die einzelnen Testungen (symptomatische Patientinnen, symptomlose Kontaktpersonen, Screening, ... ?) nicht hervor. Auch die Gesamtzahl der in diesen Altersgruppen durchgeführten Tests liegt uns nur für 3 Bundesländer bzw. Regionen vor (Tirol, Vorarlberg, Linz/Linz Land/Mühlviertel, siehe $\bullet$ Tab. 1).
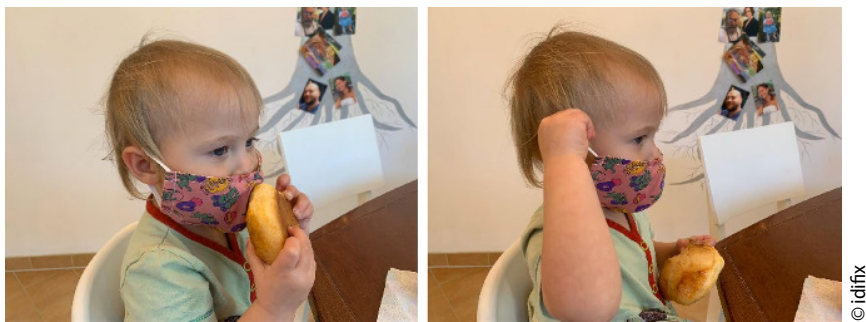
Tab. 1 Mittels SARS-CoV2 PCR getestete, positiv getestete und

stationär behandelte Kinder und Jugendliche

\begin{tabular}{|c|c|c|c|}
\hline Regionen & getestet & $\begin{array}{l}\text { positiv } \\
\text { (\%) }\end{array}$ & $\begin{array}{l}\text { stationäre } \\
\text { Behandlung }\end{array}$ \\
\hline Tirol & 2038 & $\begin{array}{l}109 \\
(5,3 \%)\end{array}$ & 2 \\
\hline Vorarlberg & 763 & $\begin{array}{l}49 \\
(6,4 \%)\end{array}$ & - \\
\hline Linz, Linz Land, Mühlviertel & 794 & $\begin{array}{l}92 \\
(11,6 \%)\end{array}$ & - \\
\hline \multicolumn{4}{|l|}{$\begin{array}{l}\text { Abteilungen für Kinder und } \\
\text { Jugendheilkunde }\end{array}$} \\
\hline Wilhelminenspital Wien & 30 & $0(0 \%)$ & $3^{\mathrm{a}}$ \\
\hline LKH Klagenfurt & - & 3 & 0 \\
\hline LKH Villach & 60 & $\begin{array}{l}1 \\
(1,7 \%)\end{array}$ & 1 \\
\hline LKH Leoben & 35 & $\begin{array}{l}1 \\
(2,8 \%)\end{array}$ & 0 \\
\hline KH Feldkirch, Bregenz, Dornbirn & 165 & $\begin{array}{l}2 \\
(1,2 \%)\end{array}$ & 2 \\
\hline LKH Eisenstadt & 210 & $0(0 \%)$ & 0 \\
\hline Univ. Klinik Wien/AKH & - & 1 & 0 \\
\hline Univ. Klinik Graz & 518 & $\begin{array}{l}3 \\
(0,6 \%)\end{array}$ & $2^{b}$ \\
\hline Univ. Klinik Innsbruck & 62 & $\begin{array}{l}2 \\
(1,7 \%)\end{array}$ & 2 \\
\hline Univ. Klinik Salzburg & 540 & $\begin{array}{l}7 \\
(1,3 \%)\end{array}$ & 0 \\
\hline Univ. Klinik Linz & 274 & $\begin{array}{l}3 \\
(1,1 \%)\end{array}$ & $4^{c}$ \\
\hline \multicolumn{4}{|l|}{$\begin{array}{l}\text { aZuweisungen von extern } \\
{ }^{\text {b} 1 ~ d a v o n ~ P I C U, ~ o h n e ~ G r u n d e r k r a n k u n g ~} \\
\text { 'eine Zuweisung von extern } \\
\text { - Daten nicht vorliegend }\end{array}$} \\
\hline
\end{tabular}

Ergänzend zu den Zahlen der Gesundheitsbehörden hat die AG Infektiologie der ÖGKJ die Daten zahlreicher Kinderabteilungen Österreichs bis Ende April erhoben (siehe - Tab. 1). In diesen Abteilungen wurden PCR Untersuchungen veranlasst, wenn PatientInnen respiratorische Symptome und/oder Fieber ohne Fokus zeigten. Nur in Einzelfällen wurden auch Kinder getestet, wenn diese aus anderen Gründen ambulant oder stationär behandelt wurden aber anamnestisch Kontaktpersonen von gesicherten SARS-CoV2 Fällen waren.

Auffallend ist, dass in den behördlich erhobenen Daten die absoluten Zahlen deutlich höher liegen als die Zahlen aus den Kinderabteilungen, die aber große Teile der pädiatrischen Bevölkerung der jeweiligen Bundesländer versorgen. Auch die relative Rate der insgesamt positiv getesteten Kinder liegt - soweit vorliegend (Tirol, Vorarlberg, Teile Oberösterreichs) - bei 5 bis $12 \%$, während die Rate der in Kinderabteilungen aufgrund von Symptomen positiv getesteten PatientInnen - selbst in den stärker betroffenen Bundesländern - bei nur 0 bis $2,8 \%$ liegt.

Diese Differenz ist wohl durch unterschiedliche Indikationen zur Testung zu erklären. Während in den Kinderabteilungen Kinder auch mit mäßig ausgeprägter respiratorischer Symptomatik und/oder (sub-)febrilen Temperaturen getestet werden, besteht für behördliche
Testungen durch das Rote Kreuz eine strengere Indikation nur bei ausgeprägter Symptomatik. Vom Roten Kreuz werden zusätzlich auch Kontaktpersonen von gesicherten SARS-CoV2 Fällen getestet, sodass diese gezielte Testung von Kindern als Kontaktpersonen die wahrscheinlichste Erklärung für die höheren Positivitätsraten in den behördlichen Angaben - verglichen mit den Daten allein aus den Kinderabteilungen - ist.

Zusammenfassend kann man sagen, dass der Anteil der Kinder an den SARS-CoV2 infizierten Personen in Österreich - wie auch international berichtet - gering ist. Die Zahl der an Kinderabteilungen aufgrund von Symptomen positiv getesteten und teilweise stationär behandelten Kinder ist noch weit geringer. Das gezielte Testen von Kindern als Kontaktpersonen scheint die plausibelste Erklärung für diesen Unterschied zu sein.

Die Rate der asymptomatisch infizierten Kinder in der Gesamtbevölkerung lässt sich aus diesen Zahlen nicht ablesen.

\section{Autoren: Volker Strenger, Christoph Zurl, Daniela Kohlfürst, Werner Zenz für die AG Infektiologie}

\author{
Dominique Sturz ${ }^{1,2, \S} \cdot$ Irene Promussas ${ }^{3} \cdot$ Thomas Kroneis $^{1,4} \cdot$ Claas \\ Röhl1,5,5 \\ ${ }^{1}$ Pro Rare Austria \\ ${ }^{2}$ Usher Initiative Austria \\ ${ }^{3}$ Lobby4Kids \\ ${ }^{4}$ KEKS Österreich \\ ${ }^{5} \mathrm{NF}$ Kinder \\ $\S$ ePAG (European Patient Advocacy Group) \\ Initiiert von Pro Rare Austria
}

\section{Statement zur Versorgung von Menschen mit seltenen und/oder chronischen Erkrankungen in Zeiten von COVID-19}

Veröffentlicht per Aussendung am 17. April 2020

\section{Einleitung}

Die COVID-19 Pandemie stellt die ganze Welt vor große Herausforderungen. Nie dagewesene, restriktive Maßnahmen sollen unser Gesundheitssystem vor einer drohenden Überlastung bewahren und die Zahl der Infektionen und Todesopfer minimieren. Ein Notbetrieb der österreichischen Kliniken, um Kapazitäten frei zu halten, ist eine Folge davon. Derzeit (Stand 7.4.) sprechen die Zahlen (Krankheitsfälle, Neuinfektionen, Genesungsrate) zwar dafür, dass wir zumindest in der ersten Welle keinen groben Engpass bei der Spitalsversorgung von PatientInnen mit COVID-19 in Österreich haben werden. Die weitere Entwicklung bleibt aber abzuwarten. Dennoch sind Universitätskliniken und Krankenhäuser weiterhin auf Notbetrieb umgestellt.

Gerade PatientInnen mit chronischen und/oder seltenen Erkrankungen sind aber auf regelmäßige Kontrollen, Behandlungen und medikamentöse und auch begleitende Therapien angewiesen. Na- 
tionale Guidelines müssen die Basisversorgung von Menschen mit seltenen und/oder chronischen Erkrankungen sicherstellen. Je länger die Ausnahmesituation andauert, desto größer ist das Risiko von gesundheitlichen Folgeschäden [1] für die Betroffenen, die in dieser Zeit unterversorgt sind. Dem offenen Brief [2] unserer europäischen Dachorganisation EURORDIS folgend, erlauben wir uns die Herausgabe dieses Statements.

\section{Versorgung von PatientInnen mit seltenen und/oder chronischen Erkrankungen}

PatientInnen mit seltenen und/oder chronischen Erkrankungen bedürfen oftmals engmaschiger Kontrollen, um sicherzustellen, dass sich ihr Gesundheitszustand nicht verschlechtert und um rechtzeitig intervenieren zu können.

Dazu zählen bildgebende Verfahren, Labordiagnostik, vom Arzt persönlich durchgeführte Untersuchungen und Eingriffe, sowie begleitende Therapien.

Je länger solche Termine aufgeschoben werden, oder Therapien nicht fortgesetzt werden, desto höher ist das Risiko von Hospitalisierungen dieser PatientInnen, mitunter auch eines steigenden Bedarfes von intensiv- medizinischer Betreuung aufgrund ihrer Grunderkrankung. Dies gilt nicht nur für PatientInnen mit seltenen Erkrankungen $[3,4]$. Ebenfalls wird das Aufholen von aufgeschobenen Kontrollen und Behandlungen nach Wiederaufnahme des Normalbetriebes lange nicht zu bewältigen sein. Es wird für manche PatientInnen also noch zu einer Verzögerung ihrer Kontrollen bis weit nach Ende der Corona-Krise kommen.

Eine kürzlich durchgeführte Umfrage unter PatientInnen mit seltenen Erkrankungen und deren Angehörigen listet einige der Probleme aber auch positive Beispiele [5].

\section{Forderungen}

- Österreichweite Guidelines zur Versorgung von Menschen mit seltenen und/oder chronischen Erkrankungen unter Beteiligung aller Stakeholder (allen voran der PatientInnenvertreterInnen)

- Sicherstellung der direkten Kommunikation zwischen Arzt/Ärztin und PatientIn

- Nutzung von vorhandenen Ressourcen (Personal, Labor, Diagnostik) innerhalb und außerhalb von Kliniken

- Sicherstellung der Begleitung Minderjähriger und beeinträchtigter Menschen, sodass diese Menschen nicht von ihren Bezugspersonen getrennt werden

\section{Triage bei Ressourcenknappheit}

Aus anderen europäischen Ländern wird von PatientInnen mit seltenen und/oder chronischen Erkrankungen berichtet, die in einer Triage-Situation benachteiligt wurden, ohne dass die Vorerkrankung mit schlechteren Heilungschancen bei COVID-19 einhergeht [6]. In Österreich muss sichergestellt werden, dass selbst bei Engpässen keine Diskriminierung dieser Gruppe stattfindet [7]. Mit dem Ausschöpfen aller Ressourcen innerhalb von Österreich oder der EU (Transfer auf Intensivstationen mit freien Kapazitäten) sollte eine Benachteiligung von Menschen mit seltenen Erkrankungen weitgehend verhindert werden können $[8,9]$.

\section{Klinische Studien in der COVID-19 Krise}

Durch die derzeitige Ausnahmesituation wurden klinische Studien ausgesetzt oder ganz eingestellt. In jenen wenigen Fällen, wo Studien fortgesetzt werden, kommt es jedoch mitunter zu einer Verschlechterung der Versorgung der PatientInnen durch eine Minimierung der Kontakte mit dem Studienpersonal. Viele PatientInnen mit einer seltenen und/oder chronischen Erkrankung profitierten bisher von der Behandlung im Rahmen einer klinischen Studie.

Mittelfristig ist die Entwicklung von Therapien für Menschen mit seltenen und oder chronischen Erkrankungen gefährdet, nicht nur durch die derzeitige Unterbrechung und Verzögerung, sondern auch durch den Fokus auf Therapieentwicklung für COVID-19 [10].

\section{Forderungen}

- Erarbeitung eines Konzeptes zur Fortsetzung der Studien unter Einbindung aller Stakeholder (Patientenorganisationen, Medizin, Behörden, pharmazeutische Industrie)

- Sicherstellung der finanziellen Ressourcen für den Betrieb von Studienzentren während und nach der Corona-Krise

\section{Sozial-, arbeitsrechtliche und bildungsrelevante Aspekte}

Ein besonders dringliches Problem stellt die derzeit nicht mögliche Freistellung von Risikopatienten mit seltenen Erkrankungen in systemrelevanten Berufen dar, ebenso fehlt diese für betreuende Eltern von Kindern, die zur Hochrisikogruppe gehören. Die (Nicht-)Aufnahme in Risikogruppen ist zudem oftmals nicht nachvollziehbar. Mit weiteren arbeits- und sozialrechtlichen Aspekten sind insbesondere der Österreichische Behindertenrat und seine Mitgliederorganisationen befasst [11].

Eine klare und eindeutige Definition der COVID-19 Risikogruppe fordert der Bundesverband Selbsthilfe Österreich [12].

\section{Best Practice Beispiele}

Einige ÄrztInnen, Fachgesellschaften, Verbände und PatientInnenorganisationen haben bereits vorbildlich und sehr schnell reagiert [11, 13]. Pro Rare Austria listet Informationen dazu auf ihrer Website [5].

\section{Literatur}

1. Hofmann J Angst vor Corona könnte zu mehr Amputationen führen. https://idwonline.de/de/news744176. Zugegriffen: 10. Apr. 2020

2. EURORDIS Recommendations to protect people living with a rare disease during the COVID-19 pandemic. https://www.eurordis.org/covid19openletter. Zugegriffen: 10. Apr. 2020

3. Lazzerini M et al (2020) Delayed access or provision of care in Italy resulting from fear of COVID-19. Lancet Child Adolesc. https://doi.org/10.1016/S23524642(20)30108-5 (Health S2352464220301085)

4. Ärztekammer für Wien Ärzte warnen vor Kollateralschäden. https://www.medinlive.at/gesundheitspolitik/aerzte-warnen-vor-kollateralschaeden (Erstellt: 15. Apr. 2020). Zugegriffen: 15. Apr. 2020

5. Sturz D, Promussas I, Kroneis T, Röhl C Mitgliederbefragung zur derzeitigen COVID-19 Krisensituation für Menschen in Österreich mit einer seltenen und/oder chronischen Erkrankung. https://www.prorare-austria.org/fileadmin/user_upload/news/2020-04-17_PRA_Fragenkatalog_COVID-19_fin.pdf (Erstellt: 17. Apr. 2020). Zugegriffen: 17. Apr. 2020

6. EURORDIS COVID-19: EURORDIS urges immediate action and proposes concrete solutions for rare disease patients. http://www.ern-rnd.eu/covid-19-eurordisurges-immediate-action-and-proposes-concrete-solutions-for-rare-diseasepatients/. Zugegriffen: 10. Apr. 2020

7. Stellungnahme der Bioethikkommission - Zum Umgang mit knappen Ressourcen in der Gesundheitsversorgung im Kontext der Covid-19- Pandemie, März 2020 (abgerufen am 17. Apr. 2020).

8. ÖGARI ICU - Triage im Falle von Ressourcen-Mangel. Empfehlungen aus Anlass der SARS-CoV-2-Pandemie. https://www.oegari.at/web_files/cms_daten/gari sop_triage_covid-19_arge_ethik_final_26.3.2020.pdf (Erstellt: 26. März 2020). Zugegriffen: 14. Apr. 2020 
9. ÖGARI Checkliste: ICU - Triage im Falle von Ressourcen-Mangel aus Anlass der SARS-CoV-2-Pandemie.https://www.oegari.at/web_files/cms_daten/gari_checkliste_triage_icu_final_26.3.2020.pdf (Erstellt: 26. März 2020).Zugegriffen: 14. Apr. 2020

10. EMA Guidance on the Management of Clinical Trials during the COVID-19 (Coronavirus) pandemic. https://ec.europa.eu/health/sites/health/files/files/eudralex/vol10/guidanceclinicaltrials_covid19_en.pdf (Erstellt: 27. März 2020).Zugegriffen: 14. Apr. 2020

11. Pichler H, Sprengseis G Ergänzungen und Änderungen im COVID-19-Gesetz. https://www.behindertenrat.at/2020/03/brief-an-bm-anschober-ergaenzungenund-aenderungen-im-covid-19-gesetz/. Zugegriffen: 10. Apr. 2020

12. BVSHOE Klare und einheitliche Definition von COVID-19 Risikogruppen notwendig. https://www.ots.at/presseaussendung/OTS_20200407_OTS0155/klare-undeinheitliche-definition-von-covid-19-risikogruppen-notwendig. Zugegriffen: 10. Apr. 2020

13. Schmidt-Erfurth U Patienteninformation. https://www.meduniwien.ac.at/hp/ augenheilkunde/. Zugegriffen: 10. Apr. 2020

\section{Coronavirus/ COVID-19 und Stillen: Aktuelle internationale Empfehlungen}

\section{Erste Version dieses Artikels: 04.03.2020, letzte Aktualisierung: 27.04.2020}

Dieser Artikel wird auf der Webseite des Europäischen Institutes für Laktation und Stillen regelmäßig aktualisiert [http://www.stillen-institut.com/de/coronavirus-covid-19-und-stillen-aktuelle-empfehlungen. html].

Glossar:

SARS-CoV2: der Erreger der aktuellen Pandemie (häufig einfach als „Coronavirus" bezeichnet, allerdings genaugenommen ein Virus, das, wie schon andere Viren zuvor, aus der Familie der Coronaviren stammt)

COVID-19: die Erkrankung, die durch Infektion mit SARS-CoV2 entsteht

Die weltweite Pandemie hat auch in den deutschsprachigen Ländern zu großen Veränderungen unseres Alltags geführt. Als medizinisches Fachpersonal begleiten wir auch Schwangere oder Stillende, die an COVID-19 erkrankt sind oder als Kontaktperson eines Infizierten gelten und somit als Verdachtsfall eingestuft werden.

In diesem Artikel finden Sie den aktuellen Stand der Empfehlungen international anerkannter Gremien und relevante Quellen, die für tiefergehende Informationen zur Verfügung stehen.

Zum Schutz des medizinischen Betreuungspersonals soll der Kontakt mit unter Verdacht stehenden oder sicher infizierten Schwangeren und Stillenden nach Maßgabe der aktuellen Empfehlungen für die Betreuung solcher Patienten erfolgen (Schutzkleidung, Isolierzimmer etc.) - Informationen dazu beim Robert-Koch-Institut (RKI) in Deutschland [https://www.rki.de/DE/Content/InfAZ/ N/Neuartiges_ Coronavirus/Hygiene.html] oder beim US-amerikanischen Center for Disease Control (CDC) [https://www.cdc.gov/coronavirus/2019$n$ CoV/hcp/index.html].

Auch wenn mehr und mehr Studien rund um das Virus weltweit veröffentlicht werden, gibt es noch immer keine ausreichende Datenbasis, um abschließende Aussagen zur speziellen Situation von Schwangeren oder Stillenden zu treffen. Aus früheren Untersuchungen zu SARS und MERS, die mit SARS-CoV2 verwandte Erreger aufweisen, werden daher international in Kombination mit den bis- herigen Erkenntnissen zur aktuellen Erkrankung vorläufige Einschätzungen vorgenommen, die stetig angepasst werden.

Wir beziehen uns in unserer untenstehenden Zusammenfassung auf folgende Quellen:

- Coronavirus Disease (COVID-19) and Breastfeeding (CDC, laufend aktualisiert) [https://www.cdc.gov/breastfeeding/breastfeeding-special-circumstances/maternal-or-infant-illnesses/covid19-andbreastfeeding.html]

- WHO Q\&A on COVID-19 an breastfeeding (WHO, 24.04.2020) [https://www.who.int/news-room/q-a-detail/q-a-on-covid-19-andbreastfeeding]

- COVID-19: Keine Angst vor Krankenhausbehandlungen oder Klinikgeburten (DGGG, 21.04.2020) [https://www.dggg.de/presse-news/pressemitteilungen/mitteilung/covid-19-keine-angst-vorkrankenhausbehandlungen-oder-klinikgeburten-1203/]

- Considerations for Inpatient Obstetric Healthcare Settings (CDC, 04.04.2020) [https://www.cdc.gov/coronavirus/2019-ncov/hcp/inpatient-obstetric-healthcare-guidance.html]

- Umgang mit Neugeborenen SARS-CoV2 positiver Mütter (DGPI, DGGG, DGPM, 31.03.2020) [https://dgpi.de/stellungnahme-dgpi-dggg-dgpm-umgang-mit-neugeborenen-sars-cov-2-positiver-muetter/]

- Women's Rights in Childbirth Must be Upheld During the Coronavirus Pandemic (ICM, 29.03.2020) [https://internationalmidwives.org/assets/files/newsfiles/2020/03/icm-statement_upholdingwomens-rights-duringcovid19-5e83ae2ebfe59.pdf]

- Leitfaden zum Umgang mit COVID-19 während Schwangerschaft und Wochenbett (OEGGG, 21.03.2020) [https://www. oeggg.at/app/download/9618663186/COVID-19\%20OEGGG-Leitfaden\%20PK-2020-03-21.pdf?t=1585506377]

- Empfohlene Präventionsmaßnahmen für die geburtshilfliche Versorgung in deutschen Krankenhäusern und Kliniken im Zusammenhang mit dem Coronavirus (DGGG, 19.03.2020) [https://www.dggg.de/fileadmin/documents/Weitere_Nachrichten/2020/COVID-19_DGGG-Empfehlungen_fuer_ Kreissaele_20200319_f.pdf]

- VSLÖ + ÖGKJ Empfehlungen Corona-Virus/COVID-19 und Stillen (VSLÖ + ÖGKJ, 14.03.2020) [https://www.stillen.at/vsloeempfehlung-corona-viruscovid-19-und-stillen/]

- Stillen und COVID-19 (Stellungnahme der Nationalen Stillkommision in Deutschland, 11.03.2020) [https://www.mri.bund.de/ de/themen/nationale-stillkommission/stellungnahmen/stillen-covid-19/]

- Statement on Coronavirus 2019 (COVID-19) (ABM, 10.03.2020, auch in deutscher Übersetzung verfügbar) [https://www.bfmed. org/abm-statement-coronavirus]

\section{Stillende Frauen (nach den ersten Tagen)}

Bisher sind keine Fälle beschrieben, bei denen der Erreger in Muttermilch gefunden wurde, eine Übertragung durch das Stillen ist daher unwahrscheinlich. Die Übertragung erfolgt typischerweise über Mund/Nase und Augen sowie möglicherweise Stuhl.

Eine stillende Mutter, die als Verdachtsfall gilt oder bestätigt mit SARS-CoV2 infiziert ist, sollte Maßnahmen treffen, um eine Übertragung auf ihr Kind zu vermeiden, ohne das Stillen zu beenden. Dazu gehört:

- gründliches Händewaschen/Desinfizieren vor und nach dem Stillen sowie vor und nach jedem Kontakt zum Kind

- Tragen eines geeigneten Mundschutzes bei jedem Kontakt mit dem Kind 
Ein Weiterstillen ist möglich. Sofern sich die Mutter gesundheitlich nicht dazu in der Lage fühlt, kann sie Muttermilch abpumpen und diese kann durch eine gesunde Betreuungsperson ohne Einschränkung verfüttert werden.

Wenn die Mutter Milch abpumpt, ist auf intensive Handhygiene zu achten, Gefäße und Pump-Sets sollten nach jedem Gebrauch sterilisiert werden.

\section{Schwangere und Peripartum-Management}

Grundsätzlich ist zu unterscheiden, ob es sich bei der Schwangeren um einen Verdachtsfall oder um eine bereits bestätigte Infektion handelt. Wenn eine bislang nicht getestete, aber unter Verdacht stehende werdende Mutter in die Geburt eintritt, ist es dringend notwendig, so rasch als möglich eine Testung und Abklärung durchzuführen. Wenn eine Infektion ausgeschlossen werden kann, bedeutet dies für Mutter, Kind und auch das betreuende Personal eine enorme Erleichterung der Situation.

Eine Übertragung durch die infizierte Schwangere auf das ungeborene Kind im Mutterleib ist unwahrscheinlich, bisher sind keine derartigen Fälle beschrieben worden. Schwangere scheinen im Grundsatz nicht häufiger oder schwerer von der Infektion betroffen zu sein als andere Bevölkerungsteile in vergleichbarem Alter und Gesundheitszustand. In früher beschriebenen Fällen (SARS und MERS) kam es allerdings häufiger zu Frühgeburten.

Der Geburtsmodus sollte anhand geburtshilflicher Indikationen und dem Wunsch der Frau individuell festgelegt werden. WHO und DGGG empfehlen, eine Sectio nur durchzuführen, wenn dies medizinisch notwendig ist, z. B. bedingt durch den Gesundheitszustand der Mutter.

Wenn eine infizierte Mutter entbindet und deshalb isoliert wird, wird im Normalfall das Kind mit ihr gemeinsam im Rooming-In verbleiben und sie wird ohne Einschränkung mit dem Stillen beginnen. In diesem Fall sollte die Mutter sorgfältig alle notwendigen HygieneMaßnahmen beachten, um die Ansteckungsgefahr für das Kind zu minimieren, insbesondere muss sie bei jedem Kontakt zum Kind einen Mundschutz tragen.

Wenn die Mutter so krank ist, dass sie sich nicht selbst um das Kind kümmern kann, sollte das Neugeborene, nach Abwägung der individuellen Situation und den vorhandenen Optionen vor Ort, temporär von ihr getrennt und als Kontaktperson eines COVID-19-Falls eingestuft werden.

Momentan beinhalten die Empfehlungen, dass es in diesem Fall bis zum Abschluss der Testung, ob es selbst infiziert ist, von gesunden Angehörigen oder medizinischem Personal ebenfalls nur in Schutzkleidung betreut werden darf. Im Interesse des Neugeborenen ist es daher wichtig, eine rasche Testung vorzunehmen.

Nachdem Muttermilch nicht selbst als Überträger des Virus beschrieben wurde, kann die Mutter bei einer Trennung ihre Muttermilch abpumpen und die Milch kann dem Baby uneingeschränkt gegeben werden. Beim Abpumpen sind besondere Hygienemaßnahmen zu beachten (s. o.). Sobald die Mutter nicht mehr ansteckend ist, kann sie zum direkten Stillen an der Brust übergehen.

\section{Verfasser: Für das Europäische Institut für Stillen und Laktation} (EISL):

Anja Bier (IBCLC), Gabriele Nindl (IBCLC), Gudrun von der Ohe (IBCLC und Ärztin)

\section{Schwangerschaft und Stillen in Zeiten von COVID19}

\author{
Fragen aus der Praxis für Gesundheitspersonal, das \\ schwangere und stillende Frauen begleitet
}

\section{Schwangerschaft}

\section{Wie sollten wir mit Routine-Terminen zur Vorsorge in der Schwangerschaft umgehen?}

Die üblichen Vorsorge-Termine in der Schwangerschaft sollten wie geplant wahrgenommen werden, sie sind medizinisch notwendig und dienen der Gesundheit von Mutter und Kind. Wenn die werdende Mutter als Verdachtsperson gilt oder sicher infiziert ist, benötigt die betreuende Fachkraft vollständige persönliche Schutzausrüstung, wie in den Verordnungen und Empfehlungen der Gesundheitsbehörden vorgeschrieben.

\section{Sind Schwangere und/oder ihr ungeborenes Kind speziell gefährdet?}

- Schwangere haben gegenüber der sonstigen Bevölkerung nach derzeitigem Stand des Wissens kein erhöhtes Risiko, sich zu infizieren und auch kein erhöhtes Risiko für einen schweren Krankheitsverlauf

- Eine Infektion der Mutter durch SARS-CoV2 während der Schwangerschaft scheint nach derzeitigem Wissensstand per se keine negativen Auswirkungen auf das Ungeborene zu haben, es infiziert sich auch nicht im Mutterleib

\section{Geburt}

\section{Wo sollten Frauen derzeit entbinden?}

Bei einer normalen Schwangerschaft ist eine ambulante Geburt im Klinikum oder Geburtshaus ebenso wie eine Hausgeburt nach wie vor möglich. Im Verdachts- oder gesicherten Infektionsfall mit SARSCoV2 gehört die Schwangere zur Risikogruppe, somit ist eine Entbindung im klinischen Setting notwendig. Zudem soll das Neugeborene $48 \mathrm{~h}$ postpartum beobachtet, aber nicht von der Mutter getrennt werden.

\section{Welcher Geburtsmodus soll im Verdachts-/Infektionsfall gewählt werden?}

Eine vaginale Geburt ist nach wie vor erste Wahl. Nach derzeitigem Wissensstand findet unter der Geburt durch den Vaginalkanal keine Infektion des Kindes statt. Wie bei jeder Entbindung (ob mit oder ohne Infektion mit SARS-CoV2) soll eine Sectio nur bei geburtshilflich-medizinischer Notwendigkeit erfolgen.

\section{Welche Maßnahmen müssen im Kreißsaal getroffen werden und} darf der Vater die Frau begleiten?

- Eine möglichst rasche Testung der unter Verdacht stehenden Mutter ist dringend notwendig, da bei negativem Ergebnis die Maßnahmen zur Isolation aufgehoben werden können, was eine große Erleichterung für Mutter, Kind und Personal mit sich bringt.

- Wenn eine Schwangere, die als Verdachtsfall gilt oder bekannt infiziert ist, in die Geburt eintritt, sollte der Kreißsaal vorab über ihre bevorstehende Ankunft informiert werden. Das gesamte betreuende Personal im Kreißsaal benötigt persönliche Schutz- 
ausrüstung, die werdende Mutter muss isoliert betreut werden. Soweit es unter der Geburt möglich ist, könnte das zusätzliche Tragen eines Mund-Nasen-Schutzes durch die Mutter hilfreich sein, um das Infektionsrisiko des Personals zu minimieren.

- Eine feste Begleitperson, die selbst symptomfrei sein muss und ebenfalls mit persönlicher Schutzausrüstung ausgestattet wird, sollte dringend unter der Geburt zugelassen werden, auch wenn für das Klinikum ansonsten ein generelles Besuchsverbot gelten mag. Für die Wochenstation legen Kliniken individuelle Regeln fest.

\section{Was passiert unmittelbar nach der Geburt?}

Auch wenn eine Mutter als Verdachtsfall eingestuft wird oder bekannt infiziert ist, sollte postpartum der normale Bonding-Prozess stattfinden, solange der Gesundheitszustand der Mutter dies zulässt. Allerdings sollte die Mutter dabei einen Mund-Nasen-Schutz (MNS) tragen. Es ist sinnvoll, auf den MNS z. B. mit Lippenstift einen Mund und Nasenlöcher aufzumalen, um das Erkennen des Gesichts-Schemas für das Neugeborene zu ermöglichen.

Das Bonding erfolgt im Optimalfall durch ungestörten direkten Hautkontakt, notwendige Untersuchungen werden auf dem Körper der Mutter durchgeführt. Das Kind erhält genügend Zeit, um selbständig zur Brust zu finden und das erste Stillen zu initiieren (durchschnittlich ca. 1 Stunde pp).

\section{Gibt etwas zu beachten, wenn eine Frau nicht stillen möchte?}

Wenn eine Mutter (unabhängig von einem möglichen Verdacht auf SARS-CoV2-Infektion) sich entschieden hat, nicht zu stillen, ist es immer wünschenswert, dass das Neugeborene auf alle Fälle Kolostrum erhalten sollte. Dies kann durch direktes Anlegen an der Brust oder durch Handgewinnung von Kolostrum geschehen. Viele Mütter, die sich gegen das Stillen entscheiden, geben ihrem Kind gerne das Kolostrum.

In der Zeit der Pandemie profitieren Neugeborene nochmals mehr von den immunologischen Eigenschaften der Muttermilch. Deshalb wäre es besonders wichtig, mit einer infizierten oder unter Verdacht stehenden Mutter darüber zu sprechen, ob sie sich vorstellen kann, für kurze Zeit zu stillen oder abzupumpen.

\section{Wochenstation}

\section{Müssen Mutter und Kind getrennt werden?}

Bei bekannter Infektion mit SARS-CoV2 bzw. bei Einstufung als Verdachtsfall werden Mutter und Kind gemeinsam isoliert und wie jeder Isolationsfall den Richtlinien der Klinik entsprechend durch die Pflege betreut.

Das Kind wird für mindestens $48 \mathrm{~h}$ postpartum beobachtet, als Verdachtsfall (Kontaktperson) eingestuft und daher nach normalem Schema so rasch als möglich getestet.

\section{Darf die Mutter stillen/darf Muttermilch verfüttert werden?}

- SARS-CoV2 wird nach derzeitigem Stand des Wissens nicht über Muttermilch übertragen. Die Mutter kann also unabhängig von ihrem Infektionsstatus ganz normal stillen. Auch in dieser Situation profitieren Neugeborene von den besonderen immunologischen Eigenschaften der Muttermilch.

- Wenn die Mutter als Verdachtsfall eingestuft wird/sicher infiziert ist, soll sie vor jedem Kontakt zum Kind die Hände desinfizieren und bei jedem Kontakt mit dem Kind einen Mund-Nasen-Schutz (MNS) tragen. Es ist sinnvoll, auf den MNS z. B. mit Lippenstift einen Mund und Nasenlöcher aufzumalen, um das Erkennen des Gesichts-Schemas für das Neugeborene zu ermöglichen.
- Wenn die Mutter in der Klinik pumpt, verbleibt die ihr zugewiesene Pumpe während der gesamten Zeit des stationären Aufenthalts bei der Mutter und es werden Einmal-Pumpsets verwendet oder die Sets werden nach jedem Gebrauch sterilisiert

\section{Was passiert, wenn die Mutter intensivpflichtig wird?}

- Bei einer an COVID-19 erkrankten intensivpflichtigen Mutter wird das Neugeborene getrennt von ihr isoliert und (anders als in anderen Fällen mit intensivpflichtiger Mutter) auch nicht zu ihr gebracht. Das Kind wird als Verdachtsfall/Kontaktperson eingestuft und nach Maßgabe für diesen Fall betreut (Neonatologie, Isolationszimmer etc.), zudem so rasch als möglich vollständig getestet.

- Wie bei jedem Kind, das von der Mutter getrennt ist, sollte alles unternommen werden, um ihm liebevollen Körperkontakt zu ermöglichen, z. B. durch einen gesunden Angehörigen in persönlicher Schutzausrüstung. Eine rasche Testung des Kindes kann die Situation deutlich erleichtern und hat daher hohe Priorität.

- Die Mutter benötigt Unterstützung für den Beginn der Milchbildung, d.h. dass das Personal sie bei der Handgewinnung von Kolostrum und beim Pump-Beginn unterstützt oder u. U. dies auch für die Frau übernimmt, bis sie wieder selbst dazu in der Lage ist - dies ist medizinisch notwendig, eine unbehandelte initiale Brustdrüsenschwellung stellt eine Komplikation dar!

\section{Wie können Mutter und Kind aus der Klinik entlassen werden?}

Wenn der Gesundheitszustand von Mutter und Kind es zulässt, werden sie im Verdachtsfall/bei Infektion der Mutter frühestens $48 \mathrm{~h}$ nach $\mathrm{Ge}$ burt und nach Maßgabe der nationalen Vorgaben und Empfehlungen der Gesundheitsbehörden in gemeinsame häusliche Quarantäne entlassen.

\section{Häuslicher Bereich}

\section{Welche Hygiene-Maßnahmen sind Zuhause zu beachten?}

- Wenn eine infizierte/unter Verdacht stehende Mutter stillt, sollte sie bis zum endgültigen negativen Test-Ergebnis/bis zur Einstufung als „nicht (mehr) infektiös“ durch die lokalen Gesundheitsbehörden vor und nach jedem Kontakt zum Kind ihre Hände desinfizieren und bei jedem Kontakt mit dem Kind einen Mund-Nase-Schutz tragen

- Wenn eine infizierte/unter Verdacht stehende Mutter für ihr Kind Muttermilch pumpt, sollte sie bis zum engültigen negativen Test-Ergebnis/bis zur Einstufung als „nicht (mehr) infektiös“ durch die lokalen Gesundheitsbehörden alle Pump-Sets, Flaschen-Sauger + Flaschen, Schnuller und sonstige Hilfsmittel nach jedem Gebrauch sterilisieren und auf gute Handhygiene achten.

\section{Was ist sonst noch wichtig?}

- Die immunologischen Eigenschaften der Muttermilch sind von großem Wert, daher ist auch ein Teil-Stillen selbstverständlich zu unterstützen

- Sobald nach einem Verdacht/einer Infektion der endgültige Negativ-Bescheid vorliegt: reduzierten Hautkontakt mit dem Kind intensiv nachholen

- Unabhängig vom Infektionsstatus gelten für alle Familien derzeit die Kontaktbeschränkungen der Gesamt-Bevölkerung: daher Besuche vermeiden, Großeltern können ihr Enkelkind vorerst nur online kennenlernen

Verfasser: Für das Europäisches Institut für Stillen und Laktation (EISL) - www.stillen-institut,com:

Anja Bier (IBCLC), Gabriele Nindl (IBCLC), Gudrun von der Ohe (IBCLC und Ärztin) 


\section{4. Österreichische Jahrestagung für Pädiatrische Psychosomatik}
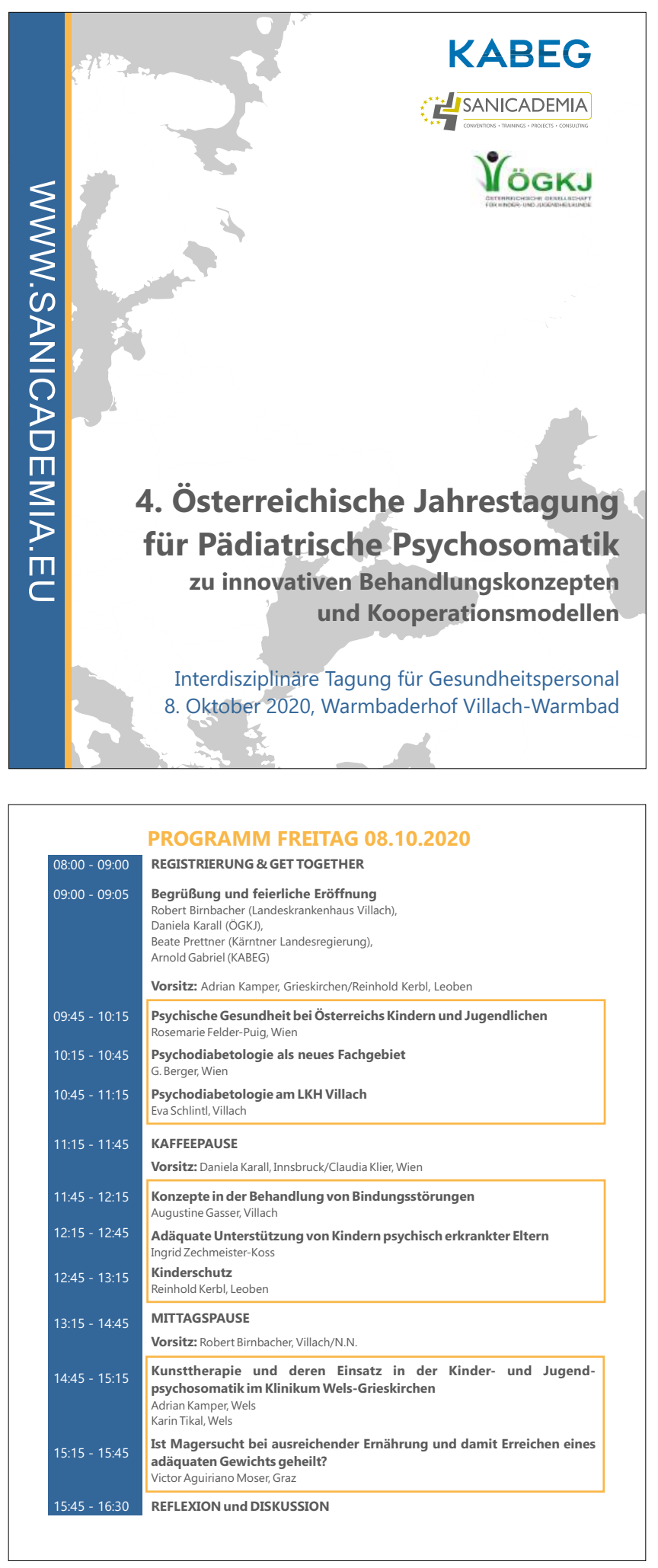

\section{Geburtstage}

Die Österreichische Gesellschaft für Kinder- und Jugendheilkunde gratuliert herzlich ihren Mitgliedern, die im nächsten Monat einen „besonderen" Geburtstag feiern:

\section{Jahre und älter werden:}

03.06.1937 | Dr. Helmuth Lothaller, Hinterbrühl

02.06.1938 | Univ. Prof. Dr. Walter Stögmann, Wien

14.06.1940 | Dr. Heinz Krisper, Wien

\section{Jahre wird:}

21.06.1950 | Dr. Hermann Josef Kahl, Düsseldorf (D)

\section{Jahre wird:}

18.06.1955 | Dr. Ute Maurer-Fellbaum, Graz

\section{Jahre werden:}

06.06.1960 | Dr. Silvana Perc-Roth, Völkermarkt

14.06.1960 | Univ. Prof. Dr. Wolfgang Schwinger, Graz

30.06.1960 | Dr. Thomas Michael Brandlhuber, Mistelbach

ÖGKJ-Geschäftsstelle Öffentlichkeitsarbeit und Standesführung

c/o S12! studio12 gmbh, Ralph Kerschbaumer

Kaiser Josef Straße 9, A-6020 Innsbruck

Tel.: +43 (0) 512-89 04 38, Fax: +43 (0) 512-89 04 38-15

E-Mail: office@paediatrie.at, www.paediatrie.at

Alle Informationen zur Anmeldung bei der ÖGKJ finden Sie unter www.paediatrie.at/mitgliedschaft. 
Hier steht eine Anzeige.

\section{Springer}

